L'Annuaire du Bureau des Longitudes pour l'année 1911. Paris, Gauthier-Villars $\left(16^{\circ}, 750\right.$ pag. Frs. 1.85$)$.

In diesem Jahre enthält das bekannte Jahrbuch außer dem gewöhnlichen astronomischen Inhalt hauptsächlich Tafeln ans dem Gebiete der Mab- und Gewichtskunde, ferner über Münzen, Geographie, Statistik und Meteorologie. Beigegeben ist ein Bericht H. Poincarés über die XVI. Konferenz von M. Bigourdan über die Sonnenfinsternis am 17. April 1912.

Physikalisch-chemische Rechenaufgaben von Dr. R. A beg g, Prof. und Dr. O. Sackur, Privatdoz. a. d. Univ. Breslau. Sammlung Göschen Nr. 445, Leipzig 1909.

52 geschickt ausgewählte Aufgaben und daran sich anschließend ihre Lösungen. Voran geht eine kurze Zusammenstellung der in den Aufgaben verwendeten Formeln. Es ist ein nützliches Hilfsmittel für den Studenten und auch für den Lehrer, der im Seminar oder sonst Aufgaben zu stellen hat.

\title{
St. $M$.
}

Die Physik in der Volksschule. Lehrproben, Entwürfe, Stoffsammlungen mit angesehlossenen Fragen und Aufgaben und Lehrplänen von Paul Jausch Duisburg. Mit 109 Abb. $384 \mathrm{~S}$. Hiezu Fragen und Aufgaben aus der Physik der Volksschule. Mit 99 Abbild. 77 S. Köln 1909, Verl. von T. P. Bachem.

Ein tapferer Versuch bereits der Volksschuljugend Freude und Verstạndnis an den physikalischen Begriffen beiznbringen. Daß dabei zaweilen über das Ziel hinausgegangen und dem kleinen Schüler Verständnis für Fragen zugemutet wird, das selbst dem großen schwer fallen mag, soll dem redlichen Bemühen, das Niveau des Physikunterrichts von Grund auf. zu heben, zu gute gehalten werden.

St. $M$.

Aerodynamik von F. W. Lanchester. Ein Gesamtwerk über das Fliegen. Aus dem Englischen iubersetzt von C. und A. Runge in Göttingen. Erster Band. Mit Anhängen über dio Geschwindigkeit und den Impuls von Schallwellen, ubber die Theorie des Segelfluges n. s. w. Mit 162 Fig. und 1 Tafel XIV + 344 S. Verl. B. G. Teubner, Leipzig und Berlin 1909. Preis M. 12.

Dieses, das modernste Gebiet nmfassende Werk ist bereits 1907 entstanden, also hinter den Erfolgen der praktischen Aviatik scheinbar etwas zurück. Aber es enthält alle sicher fandierten Grundsätze, die der Theorie bisher zugänglich sind, in geradezu musterhafter, klarer Darstellung. Der deutschen Aasgabe 1909 setzt C. Runge die Worte voran: „Das vorliegende Werk enthält so viele für die Entwicklung des freien Fluges wichtige originelle Ideen and Ausführungen, daB die deutsehen Ingenieure and Gelehrten es der Verlagshandlung Dank wissen werden, eine Übersetzung veranstaltet zu haben . . . . F Für die Gelehrten liegt der Hauptreiz des Buches in den Ideen utber Flüssigkeitsreibung, die in klarer mathematischer Formulierung herauszuarbeiten, die nächste Aufgabe der Hydrodynamik sein durfte." Das Werk behandelt in zehn Kapiteln: Widerstand in einer Flüssigkeit und verwandte Erseheinungen; 
Zähigkeit und Oberflachenreibung; die Hydrodynamik der analytischen Theorie; Flügelform und Bewegnng in der Perjpterie; Aeroplan, senkrechte Fläche; der geneigte Aeroplan; die Ökonomie des Flnges; das Aerophyll; über die Triebkraft, den Schraubenpropeller und die beim Flug verausgabte Energie; die experimentelle Aerodynamik.

Berücksichtigung der grobartigen Erfolge der jüngsten Zeit wären im II. Teil zu erhoffen, auf dessen Erscheinen man billigermaßen gespannt sein darf.

St. $M$.

Lehrbuch der Physik, zum Gebrauche beim Unterricht, bei akademischen Vorlesungen und zum Selbststudium von E. Grimsehl. B. G. Teubner, Leipzig \& Berlin 1909. 1052 Seiten, 1091 Figuren und 2 farbige Tafeln. Geb. M. 16.

Aus dem Unterricht an der Oberrealschule hervorgegangen ist dieses Buch doch weit mehr als ein Mittelschullehrbuch und wohl geeignet, den dreifachen, im Titel genannten Zweck zu erfüllen. Elementar gehalten - nur an einzelnen Stellen wird von den Elementen der höheren Mathematik Gebrauch gemacht - zeichnet es sich vor anderen ähnlichen Werken besonders durch die große Zahl einfacher und instruktiver Schulversuche aus, durch die die Grundsätze der einzelnen Kapitel veranschaulicht werden und die das Buch namentlich dem Lehrer empfehlen. Auch die jüngsten Gebiete, wie die Radioaktivität, werden behandelt, doch hat sich der Verfasser hier offenbar aus Furcht, unsicheres in sein Lehrbuch anfzunehmen, groRe Zurückbaltung anferlegt. Wir vermissen eine Angabe über die Größe des Elementarquantums und den Namen J. J. Thomson. Ein Anhang enthält Tabellen wichtiger physikalischer Konstanten und mathematische Tafeln.

K. Prz.

Les bases physico-chimiques de la chimie analytique par le Dr. W. Herz. Übersetzt von E. Philippi. Paris, Gauthier-Villars, 1909, 167 Seiten.

Der Zweck dieses Buches, das als ein Band der Monographiensammlung "L'analyse Chimique" von Dr. P. M. Margosches erscheint, ist es, den analytischen Chemiker über die seinen Methoden za Grunde liegenden physikalischchemischen Prinzipien aufzuklären. Klar und einfach geschrieben, kann es überhaupt als Einführung in die physikalische Chemie empfoblen werden.

K. Prz.

Grundlagen der Analysis von. M. Pas ch, ausgearbeitet unter Mitwirkung von Cl. Thaer. Leipzig u. Berlin, B. G. Teubner, 1908; VI. u. 140 S.; Preis geh. M. 3.60, geb. M. 4.-.

Die Verdienste, die sich Pasch um die Erforschung der Grundlagen der Geometrie erworben hat, insbesondere durch seine „Vorlesungen über neuere Geometrie," sind zu bekannt, als daß daran erinnert werden müßte. Wir freuen uns, hier ein Buch anzeigen zu können, in dem nun Pasch auch seine Gedanken über die Grundlagen der Analysis zusammenfabt, wir freuen uns darüber umso mehr, als diese Gedanken durchaus origineller Natur sind, und sich von den bekannten, und mehr oder weniger anerkannten anderen Darstellungen der Grundlagen der Arithmetik teilweise sehr weit unterscheiden. Der Standpunkt, den der Verfasser einnimmt, ist derselbe, den er in den erwähnten "Vorlesungen über neuere Geometrie" vertreten hat; es dürfte zu seiner Charakterisierung am zweckmäßigsten sein, einige Sätze des einleiten- 